Article

\title{
Ideal Convergence and Completeness of a Normed Space
}

\author{
Fernando León-Saavedra ${ }^{1}$, Francisco Javier Pérez-Fernández ${ }^{2}$, \\ María del Pilar Romero de la Rosa ${ }^{3, *}$ and Antonio Sala ${ }^{4}$ \\ 1 Department of Mathematics, Faculty of Social Sciences and Communication, University of Cádiz, \\ 11405 Jerez de la Frontera, Spain; fernando.leon@uca.es \\ 2 Department of Mathematics, Faculty of Science, University of Cádiz, 1510 Puerto Real, Spain; \\ javier.perez@uca.es \\ 3 Department of Mathematics, CASEM, University of Cádiz, 11510 Puerto Real, Spain \\ 4 Department of Mathematics, Escuela Superior de Ingeniería, University of Cádiz, 11510 Puerto Real, Spain; \\ antonio.sala@uca.es \\ * Correspondence: pilar.romero@uca.es
}

Received: 23 July 2019; Accepted: 21 September 2019; Published: 25 September 2019

\begin{abstract}
We aim to unify several results which characterize when a series is weakly unconditionally Cauchy (wuc) in terms of the completeness of a convergence space associated to the wuc series. If, additionally, the space is completed for each wuc series, then the underlying space is complete. In the process the existing proofs are simplified and some unanswered questions are solved. This research line was originated in the $\mathrm{PhD}$ thesis of the second author. Since then, it has been possible to characterize the completeness of a normed spaces through different convergence subspaces (which are be defined using different kinds of convergence) associated to an unconditionally Cauchy sequence.
\end{abstract}

Keywords: ideal convergence; unconditionally Cauchy series; completeness ; barrelledness

MSC: 40H05; 40A35

\section{Introduction}

A sequence $\left(x_{n}\right)$ in a Banach space $X$ is said to be statistically convergent to a vector $L$ if for any $\varepsilon>0$ the subset $\left\{n:\left\|x_{n}-L\right\|>\varepsilon\right\}$ has density 0 . Statistical convergence is a summability method introduced by Zygmund [1] in the context of Fourier series convergence. Since then, a theory has been developed with deep and beautiful results [2] by different authors, and moreover at the present time this theory does not present any symptoms of abatement. The theory has important applications in several branches of Applied Mathematics (see the recent monograph by Mursaleen [3]).

It is well known that there are results that characterize properties of Banach spaces through convergence types. For instance, Kolk [4] was one of the pioneering contributors. Connor, Ganichev and Kadets [5] obtained important results that relate the statistical convergence to classical properties of Banach spaces.

The aim of this paper originates in the PhD thesis of the second author [6] who discovered a relationship between properties of a normed space $X$ and some sequence spaces which are called convergence spaces associated to a weakly unconditionally Cauchy series. Notice that these sequence spaces associated to a weakly unconditionally Cauchy series (in brief wuc series) were defined originally [6] in terms of the norm topology and the usual weak topology of the space. Since then, these kinds of results have been investigated in several convergence spaces associated with a weakly unconditionally Cauchy series using different types of convergence [7-12]. In fact, several questions 
remain unsolved for different kinds of convergence. For instance, $A$-statistical convergence and $A$-strong convergence (where $A$ is a non-negative matrix) were introduced by Connors in [13]. Theorem 3.5 in [12] remains open for $p \in(0,1)$ and Theorems 3.1, 3.5 and 5.1 in [12] remains unsolved in the $A$-statistical convergence setting.

In this paper we aim to unify some known results. In the process we pull together much of what is known about this topic and we will simplify some of their existing proofs. As a consequence we provide an unified point of view which allows us to solve several unsolved questions. In fact, we will obtain results in the context of ideal convergence. We will show that under reasonable conditions on a given non-trivial ideal, the studied properties do not depend on the ideal that we use to define the convergence spaces associated to the wuc series. This allows us to extend our results for an arbitrary summability method that shares some kind of ideal-convergence on the realm of all bounded sequences. This will allow us to unify the known results and obtain answers to some unresolved questions.

The paper is organized as follows. In Section 2, we will study the convergence induced by an ideal $\mathcal{I} \subset \mathcal{P}(\mathbb{N})$, (that is, the $\mathcal{I}$-convergence), which will provide the general framework of our results in Section 3. Next we will review some basic properties and some preliminary results about $\mathcal{I}$-convergence that we will use later. Section 3 deals with the space of $\mathcal{I}$-summability (which we will denote by $S_{\mathcal{I}}\left(\sum_{i} x_{i}\right)$ ) associated to a weakly unconditionally Cauchy series $\sum_{i} x_{i}$. It is shown that for any non-trivial regular ideal $\mathcal{I}$, a series $\sum_{i} x_{i}$ is weakly unconditionally Cauchy if and only if $S_{\mathcal{I}}\left(\sum_{i} x_{i}\right)$ is complete. Moreover, if this equivalence is true for each series in a normed space $X$, then the space $X$ must be complete. There is a counterpart of the above results for the weak topology, and moreover, we were able to extend these results for certain general summability methods. Finally, for the $w^{*}$-topology of $X$ we will characterize when a series $\sum_{i} f_{i}$ in the dual space $X^{*}$ is wuc, and this characterization incorporates general summability methods. Moreover, this result is sharpened when the space $X$ is barrelled. The paper concludes with a brief section on applications.

\section{Some Preliminary Results}

Next we will see the general framework where we will prove our results. Let $(X, d)$ be a metric space. A summability method $\rho$ on $X$ is a rule to assign limits to a sequence, that is, it is a map $\rho: D_{\rho} \subset X^{\mathbb{N}} \rightarrow X$. A summability method $\rho$ is said to be regular if for each convergent sequence $\left(x_{n}\right)$ in $X$, that is, $\lim _{n \rightarrow \infty} x_{n}=x_{0}$, we have that $\rho\left(\left(x_{n}\right)\right)=x_{0}$.

Let us denote by $\mathcal{P}(\mathbb{N})$ the power set of $\mathbb{N}$. Let us consider $\mathcal{I} \subset \mathcal{P}(\mathbb{N})$ an arbitrary family of subsets of $\mathbb{N}$. We will say that $\mathcal{I}$ is a non-trivial ideal if

1. $\mathcal{I} \neq \varnothing$ and $\mathcal{I} \neq \mathcal{P}(\mathbb{N})$.

2. If $A, B \in \mathcal{I}$ then $A \cup B \in \mathcal{I}$.

3. If $A \subset B$ and $B \in \mathcal{I}$ then $A \in \mathcal{I}$.

4. Additionally we say that $\mathcal{I}$ is regular (or admissible) if it contains all finite subsets.

We will say that a sequence $\left(x_{n}\right) \subset X$ is $\mathcal{I}$-convergent to $L$ (in short we wil denote $L=$ $\mathcal{I}-\lim _{n \rightarrow \infty} x_{n}$ or $\left.x_{n} \stackrel{\mathcal{I}}{\longrightarrow} L\right)$ if for any $\varepsilon>0$ the subset

$$
A(\varepsilon)=\left\{n \in \mathbb{N}: d\left(x_{n}, L\right)>\varepsilon\right\} \in \mathcal{I}
$$

Let us observe that if $\mathcal{I}$ is the set of all finite subsets of $\mathbb{N}$ then we recover the usual convergence. And if $\mathcal{I} \subset \mathcal{J}$ then $\mathcal{I}$-convergence implies $\mathcal{J}$-convergence. In particular, usual convergence implies $\mathcal{I}$-convergence for a regular ideal. Thus, eventually constant sequences $\mathcal{I}$-converge for a regular ideal $\mathcal{I}$.

In general, uniqueness of the limit is not true for $\mathcal{I}$-convergence. However when $\mathcal{I}$ is non-trivial, then an ideal $\mathcal{I}$ defines a summability method. Essentially, we need to show that the limit, when it exists, is unique. 
Proposition 1. Let $X$ be a metric space and let $\mathcal{I}$ be a non-trivial ideal. Suppose that for a sequence $\left(x_{n}\right)$ we have $\mathcal{I}$ - $\lim _{n \rightarrow \infty} x_{n}=L_{1}$ and $\mathcal{I}$ - $\lim _{n \rightarrow \infty} x_{n}=L_{2}$, then $L_{1}=L_{2}$.

Proof. Suppose on the contrary that $L_{1} \neq L_{2}$. Let $\varepsilon>0$ be such that $B\left(L_{1}, \varepsilon\right) \cap B\left(L_{2}, \varepsilon\right)=\varnothing$. Since $\mathcal{I}$ - $\lim _{n \rightarrow \infty} x_{n}=L_{i}, i=1,2$, we have that:

$$
C_{1}=\left\{n: x_{n} \notin B\left(L_{1}, \varepsilon\right)\right\} \in \mathcal{I}
$$

and

$$
C_{2}=\left\{n: x_{n} \notin B\left(L_{2}, \varepsilon\right)\right\} \in \mathcal{I} .
$$

That is, $C_{1} \cup C_{2}=\mathbb{N} \in \mathcal{I}$, a contradiction.

We will say that sequence $\left(x_{n}\right)$ is $\mathcal{I}$-Cauchy if for each $\varepsilon>0$ there exists $k \in \mathbb{N}$ such that the subset $\left\{n \in \mathbb{N}: d\left(x_{n}-x_{k}\right) \geq \varepsilon\right\} \in \mathcal{I}$.

The following result is an extension of Fridy's result [14], that was proved in [15] and it will be used later.

Theorem 1 (Dems). Assume that $\mathcal{I} \subset \mathcal{B}(\mathbb{N})$ is a non-trivial ideal.

(i) If $X$ is a complete metric space then every $\mathcal{I}$-Cauchy sequence in $X$ is $\mathcal{I}$-convergent in $X$.

(ii) Assume that $\mathcal{I}$ is a no-trivial regular ideal. Then, if every $\mathcal{I}$-Cauchy sequence in $X$ is $\mathcal{I}$-convergent in $X$ then $\mathrm{X}$ must be complete.

Now if $X$ is a normed space, given a non-trivial ideal $\mathcal{I}$, analogously we can define the weak- $\mathcal{I}$ convergence for a sequence $\left(x_{n}\right)_{n}$. A sequence $\left(x_{n}\right)_{n}$ is said to be weak- $\mathcal{I}$ convergent to $L \in X$ (in short $w \mathcal{I}$ - $\left.\lim _{n \rightarrow \infty} x_{n}=L\right)$ if for any $f \in X^{*}$ and for any $\varepsilon>0$ the subset $\left\{n:\left|f\left(x_{n}\right)-f(L)\right| \geq \varepsilon\right\} \in \mathcal{I}$. Let us observe that a sequence $\left(x_{n}\right) \subset X$ is weak-I-I-convergent to $L \in X$ if and only if for every weak-neighbourhood $U$ of $L$ the set $\left\{n \in \mathbb{N}: x_{n} \notin U\right\}$ belongs to $\mathcal{I}$.

Clearly we have:

Proposition 2. Assume that $\mathcal{I}$ is a non-trivial ideal. If $\mathcal{I}$ - $\lim _{n \rightarrow \infty} x_{n}=L$ then $w \mathcal{I}$ - $\lim _{n \rightarrow \infty} x_{n}=L$.

The following result is a simple test to show that a sequence $\left(x_{n}\right)$ has no weak- $\mathcal{I}$ limit.

Proposition 3. Assume that $\mathcal{I}$ is a non-trivial regular ideal. Suppose that for a sequence $\left(x_{n}\right)$ there exists $f \in X^{*}$ such that $\lim _{n \rightarrow \infty} f\left(x_{n}\right)=\infty$ then $\left(x_{n}\right)$ does not weak $\mathcal{I}$-converge to any $L \in X$.

Proof. Let $f \in X^{*}$ such that $\lim _{n \rightarrow \infty} f\left(x_{n}\right)=\infty$. Then, given $\varepsilon>0$, the subset

$$
\left\{n:\left|f\left(x_{n}\right)-f(L)\right| \geq \varepsilon\right\} \in \mathcal{I}
$$

has a finite complement. Hence, since $\mathcal{I}$ is regular, $\mathbb{N} \in \mathcal{I}$, a contradiction.

\section{Main Results}

Throughout this section $X$ will denote a real normed space. (The main results of this section can be easily recovered for the complex case.) A series $\sum_{i} x_{i}$ in a normed space $X$ is said to be weakly unconditionally Cauchy (wuc) if $\sum_{i}\left|f\left(x_{i}\right)\right|<\infty$ for each $f \in X^{*}$, or equivalently $\left\{\sum_{i=1}^{n} a_{i} x_{i}:\left(a_{i}\right) \in\right.$ $\left.B_{\ell}, n \in \mathbb{N}\right\}$ is bounded in the normed space $X$.

Let $\sum_{i} x_{i}$ be a series in a Banach space $X$, let us define

$$
S_{\mathcal{I}}\left(\sum_{i} x_{i}\right)=\left\{\left(a_{i}\right) \in \ell_{\infty}: \sum_{i=1}^{n} a_{i} x_{i} \text { is } \mathcal{I} \text {-convergent }\right\}
$$


endowed with the supremum norm. This space will be called the $\mathcal{I}$-convergence space associated to the series $\sum_{i} x_{i}$. The following result characterizes the completeness of the space $S_{\mathcal{I}}\left(\sum_{i} x_{i}\right)$.

Theorem 2. Let $X$ be a Banach space and let $\mathcal{I} \subset \mathcal{P}(\mathbb{N})$ be a non-trivial regular ideal. The following conditions are equivalent:

1. $\sum_{i} x_{i}$ is a weakly unconditionally Cauchy series (wuc).

2. $S_{\mathcal{I}}\left(\sum_{i} x_{i}\right)$ is a complete space.

3. $c_{0} \subset S_{\mathcal{I}}\left(\sum_{i} x_{i}\right)$.

Proof. Let us show that $(1) \Rightarrow(2)$. Since $\sum x_{i}$ is wuc, the following supremum is finite:

$$
H=\sup \left\{\left\|\sum_{i=1}^{n} a_{i} x_{i}\right\|:\left|a_{i}\right| \leq 1,1 \leq i \leq n, n \in \mathbb{N}\right\}<+\infty .
$$

Let $\left(a^{m}\right) \subset S_{\mathcal{I}}\left(\sum_{i} x_{i}\right)$ such that $\lim _{m}\left\|a^{m}-a^{0}\right\|_{\infty}=0$, with $a^{0} \in \ell_{\infty}$. We wish to prove that $a^{0} \in S_{\mathcal{I}}\left(\sum_{i} x_{i}\right)$, that is, there exists $L \in X$ such that $L=\mathcal{I}$ - $\lim S_{n}^{0}$, where $S_{n}^{0}=\sum_{j=1}^{n} a_{j}^{0} x_{j}$. According to Theorem 1 (ii), since $\mathcal{I}$ is non-trivial, regular and $X$ is complete, the sequence $S_{n}^{0}$ is $\mathcal{I}$-convergent if and only if it is $\mathcal{I}$-Cauchy. Thus, we want to show that for any $\varepsilon>0$ there exists $k_{0} \in \mathbb{N}$ such that the subset

$$
A_{0}\left(\varepsilon, k_{0}\right)=\left\{n:\left\|S_{n}^{0}-S_{k_{0}}^{0}\right\| \geq \varepsilon\right\} \in \mathcal{I} .
$$

On the other hand, since $\lim _{m}\left\|a^{m}-a^{0}\right\|_{\infty}=0$, there exists $m_{0} \in \mathbb{N}$ such that for all $m \geq m_{0}$. $\left\|a^{m}-a^{0}\right\| \leq \frac{\varepsilon}{4 H}$. Hence, we have

$$
\left\|\sum_{i=1}^{j} \frac{4 H}{\varepsilon}\left(a_{i}^{m}-a_{i}^{0}\right) x_{i}\right\| \leq H,
$$

therefore, we obtain that for all $m \geq m_{0}$ and for all $j \in \mathbb{N}$,

$$
\left\|S_{j}^{m}-S_{j}^{0}\right\| \leq \frac{\varepsilon}{4} .
$$

Now, let us observe that since $a^{m_{0}} \in S_{\mathcal{I}}$, we have that $\left(S_{j}^{m_{0}}\right)$ is $\mathcal{I}$-convergent. On the other hand, since $\mathcal{I}$ is regular we have equivalently that $\left(S_{j}^{m_{0}}\right)$ is a $\mathcal{I}$-Cauchy. Thus, for each $\varepsilon>0$ there exists $k_{0} \in \mathbb{N}$ such that the subset

$$
A_{m_{0}}\left(\varepsilon, k_{0}\right)=\left\{n \in \mathbb{N}:\left\|S_{n}^{m_{0}}-S_{k_{0}}^{m_{0}}\right\| \geq \varepsilon\right\} \in \mathcal{I} .
$$

Thus, for this natural number $k_{0}$, if $n \in A_{0}\left(\varepsilon, k_{0}\right)$ we have that

$$
\begin{aligned}
\left\|S_{n}^{m_{0}}-S_{k_{0}}^{m_{0}}\right\| & =\left\|S_{n}^{m_{0}}-S_{k_{0}}^{m_{0}}-S_{n}^{0}+S_{n}^{0}+S_{k_{0}}^{0}-S_{k_{0}}^{0}\right\| \\
& =\left\|S_{n}^{0}-S_{k_{0}}^{0}+S_{n}^{m_{0}}-S_{n}^{0}+S_{k_{0}}^{0}-S_{k_{0}}^{m_{0}}\right\| \\
& \geq \varepsilon-\frac{\varepsilon}{4}-\frac{\varepsilon}{4}=\frac{\varepsilon}{2} .
\end{aligned}
$$

Thus, if $n \in A_{0}\left(\varepsilon, k_{0}\right)$ then $n \in A_{m_{0}}\left(\varepsilon / 2, k_{0}\right)$, therefore $A_{0}\left(\varepsilon, k_{0}\right) \subset A_{m_{0}}\left(\varepsilon / 2, k_{0}\right)$. Since $A_{m_{0}}\left(\varepsilon / 2, k_{0}\right) \in \mathcal{I}$ we obtain that $A_{0}\left(\varepsilon, k_{0}\right) \in \mathcal{I}$ as we desired.

Now, let us observe that if $S_{\mathcal{I}}\left(\sum_{i} x_{i}\right)$ is a complete space, it contains the space of eventually zero sequences $c_{00}$ and therefore we have (2) $\Rightarrow(3)$. 
Finally, let us show (3) $\Rightarrow(1)$. If the series $\sum x_{i}$ is not wuc, there exists $f \in X^{*}$ such that $\sum_{i=1}^{\infty}\left|f\left(x_{i}\right)\right|=+\infty$. Inductively, we will construct a sequence $\left(a_{i}\right)_{i} \in c_{0}$ such that $\sum_{i} a_{i} f\left(x_{i}\right)=+\infty$ and $a_{i} f\left(x_{i}\right) \geq 0$.

Since $\sum_{i=1}^{\infty}\left|f\left(x_{i}\right)\right|=+\infty$, there exists $m_{1}$ such that $\sum_{i=1}^{m_{1}}\left|f\left(x_{i}\right)\right|>2 \cdot 2$. We define $a_{i}=\frac{1}{2}$ if $f\left(x_{i}\right) \geq 0$ and $a_{i}=-\frac{1}{2}$ if $f\left(x_{i}\right)<0$ for $i \in\left\{1,2, \ldots, m_{1}\right\}$. This implies that $\sum_{i=1}^{m_{1}} a_{i} f\left(x_{i}\right)>2$ and $a_{i} f\left(x_{i}\right) \geq 0$ if $i \in\left\{1,2, \ldots, m_{1}\right\}$.

Let $m_{2}>m_{1}$ be such that $\sum_{i=m_{1}+1}^{m_{2}}\left|f\left(x_{i}\right)\right|>2^{2} \cdot 2^{2}$. We define $a_{i}=\frac{1}{2^{2}}$ if $f\left(x_{i}\right) \geq 0$ and $a_{i}=-\frac{1}{2^{2}}$ if $f\left(x_{i}\right)<0$ for $i \in\left\{m_{1}+1, \ldots, m_{2}\right\}$. Then, $\sum_{i=m_{1}+1}^{m_{2}} a_{i} f\left(x_{i}\right)>2^{2}$ and $a_{i} f\left(x_{i}\right) \geq 0$ if $i \in\left\{m_{1}+1, \ldots, m_{2}\right\}$.

Inductively we obtain a sequence $\left(a_{i}\right)_{i} \in c_{0}$ for which $\sum_{i=1}^{n} a_{i} f\left(x_{i}\right) \rightarrow \infty$. Then by Proposition 3 we have that $\sum_{i=1}^{n} a_{i} x_{i}$ does not $\mathcal{I}$-converge to any $L \in X$. Therefore $\left(a_{i}\right) \notin S_{\mathcal{I}}\left(\sum_{i} x_{i}\right)$, a contradiction.

Remark 1. For future references, let us observe that in the proof of Theorem 2, the completeness is used only in the implication $(1) \Rightarrow(2)$.

Next, we will try to describe a general frame for Theorem 2. We wish to extend Theorem 2 for a general convergence method $\rho$. However, we need to place some limits on the properties of $\rho$.

Definition 1. Let $\rho: D_{\rho} \subset X^{\mathbb{N}} \rightarrow X$ be a summability method on a metric space $X$. We will say that $\rho$ is a natural summability method if there exists a non-trivial regular ideal $\mathcal{I} \subset \mathcal{P}(\mathbb{N})$ such that

1. If $x_{n} \stackrel{\rho}{\longrightarrow} x$ then $x_{n} \stackrel{\mathcal{I}}{\longrightarrow} x$.

2. If $\left(x_{n}\right)$ is bounded and $x_{n} \stackrel{\mathcal{I}}{\longrightarrow} x$, then $x_{n} \stackrel{\rho}{\longrightarrow} x$. That is, in the realm of all bounded sequences the convergence method $\rho$ is equivalent to the summability method induced by a non-trivial regular ideal $\mathcal{I}$.

Remark 2. Let us mention that the above situation occurs very often. For instance, a classical example of natural summability method is the strong $\mathrm{w}^{p}$-Cesàro summability $(0<p<\infty)$, thanks to a beautiful result by Connor [16]. Let us recall that a sequence $\left(x_{n}\right)_{n} \subset X$ is strong $\mathrm{w}^{p}$-convergent to $L \in X$ if $\lim _{n \rightarrow \infty} \frac{1}{n} \sum_{i=1}^{n}\left\|x_{n}-L\right\|^{p}=0$. Connor established in [16], that if a sequence is strong $\mathrm{w}^{p}$-convergent then it is statistical convergent. Moreover, in the realm of all bounded sequences both convergence methods are equivalent. It is also well known that statistical convergence is a special case of ideal convergence.

Given a convergence method $\rho$ for series in a normed space $X$ we define abstractly the $\rho$-sequence space associated to a series $\sum_{i} x_{i}$ as follows:

$$
S_{\rho}\left(\sum x_{i}\right)=\left\{\left(a_{i}\right) \in \ell^{\infty}: \sum_{i=1}^{n} a_{i} x_{i} \text { is } \rho \text {-convergent in } X\right\} .
$$

The proof of the following Corollary follows essentially the ideas of Theorem 2 and it is essentially trivial using the equivalence between $\rho$-convergence and ideal convergence for bounded series.

Corollary 1. Let X be a Banach space and let $\rho$ a natural summability method. Then, the following conditions are equivalent:

1. $\sum_{i} x_{i}$ is a weakly unconditionally Cauchy series (wuc).

2. $S_{\rho}\left(\sum_{i} x_{i}\right)$ is a complete space.

3. $c_{0} \subset S_{\rho}\left(\sum_{i} x_{i}\right)$.

Theorem 3. Let $\mathcal{I} \subset \mathcal{P}(\mathbb{N})$ be a non-trivial regular ideal. Then a normed space $X$ is complete if and only if $S_{\mathcal{I}}\left(\sum_{i} x_{i}\right)$ is closed in $\ell^{\infty}$ for each wuc series $\sum_{i} x_{i}$.

Proof. If $X$ is complete then Theorem 2 shows that $S_{\mathcal{I}}\left(\sum_{i} x_{i}\right)$ is complete for each wuc series $\sum_{i} x_{i}$. 
Conversely, let us suppose that $X$ is not complete. Then, there exists a series $\sum_{i} x_{i}$ with $\left\|x_{i}\right\| \leq \frac{1}{i 2^{i}}$ such that $\sum_{i} x_{i}=x^{* *} \in X^{* *} \backslash X$. Since $X^{* *}$ is a Banach space with the dual topology, if $S_{n}=\sum_{i=1}^{n} x_{i}$ then $\sup _{\left\|y^{*}\right\| \leq 1}\left|y^{*}\left(S_{n}\right)-x^{* *}\left(y^{*}\right)\right| \rightarrow 0$, that is, $\sum_{i} y^{*}\left(x_{i}\right)=x^{* *}\left(y^{*}\right)$, for all $\left\|y^{*}\right\| \leq 1$.

Set $y_{i}=i x_{i}$. Since $\left\|y_{i}\right\| \leq \frac{1}{2^{i}}$, we obtain that $\sum_{i} y_{i}$ is absolutely convergent, hence $\sum_{i} y_{i}$ is a wuc series. We claim that $\sum_{i=1}^{n} \frac{1}{i} y_{i}$ is not $\mathcal{I}$-convergent in $X$. On the contrary, let us suppose that $\sum_{i=1}^{n} \frac{1}{i} y_{i}$ is $\mathcal{I}$-convergent to $L \in X$. Thus, according to Proposition 2 for each $y^{*} \in X^{*}$ with $\left\|y^{*}\right\| \leq 1$, we have that $\sum_{i=1}^{n} \frac{1}{i} y^{*}\left(y_{i}\right) \mathcal{I}$-converges to $y^{*}(L)$. Therefore, according to Proposition 1 , we have the uniqueness of the limit, that is, $x^{* *}\left(y^{*}\right)=y^{*}(L)$ for all $\left\|y^{*}\right\| \leq 1$. Hence $x^{* *}=L \in X$, a contradiction. Therefore $\sum_{i=1}^{n} \frac{1}{i} y_{i}$ is not $\mathcal{I}$ convergent to any $L \in X$. Finally, since $\sum_{i} y_{i}$ is wuc and $\sum_{i=1}^{n} \frac{1}{i} y_{i}$ is not $\mathcal{I}$ convergent we have that $\left(\frac{1}{n}\right) \in c_{0}$ but $\left(\frac{1}{n}\right) \notin S_{\mathcal{I}}\left(\sum_{i} x_{i}\right)$ which is a contradiction with (3) in Theorem 2 (see also Remark 1), and the proof is complete.

Let us observe that the opposite implication of the next Corollary does not use the naturality of $\rho$ in its full generality, thus for the converse implication, it is possible to refine the hypothesis; basically we need only to guarantee the uniqueness of some limits.

Corollary 2. Let $\rho$ be a natural summability method. A normed space $X$ is complete if and only if $S_{\rho}\left(\sum_{i} x_{i}\right)$ is closed in $\ell^{\infty}$ for each wuc series $\sum_{i} x_{i}$.

Proof. The first implication follows directly using Corollary 1 . For the converse implication, let $\mathcal{I}$ be the ideal which guarantees the naturality of $\rho$. Let us suppose that $X$ is not complete, then using the construction made in Theorem 3 there exists $\left(x_{n}\right)$, with $\left\|x_{n}\right\| \leq \frac{1}{n 2^{n}}$, such that $\sum x_{n}=x^{* *} \in X^{* *} \backslash X$ which implies that $\sum_{n} y^{*}\left(x_{n}\right)=x^{* *}\left(y^{*}\right)$ for all $\left\|y^{*}\right\| \leq 1$.

If we consider the series $\sum_{n} y_{n}$ where $y_{n}=n x_{n}$, we have that $\sum_{n} y_{n}$ is wuc. We claim that $S_{n}=\sum_{i=1}^{n} \frac{1}{i} y_{i}$ does not $\rho$-converge to any $L \in X$. Suppose on the contrary that $S_{n} \stackrel{\rho}{\longrightarrow} L$, since $\left\{S_{n}\right\}$ is bounded we have $S_{n} \stackrel{\mathcal{I}}{\longrightarrow} L$. But the last assertion is not possible, according to Proposition 1, for all $y^{*} \in X^{*}$, we have that $y^{*}(L)=x^{* *}\left(y^{*}\right)$, that is $L=x^{* *} \in X$. Therefore, $S_{n}=\sum_{i} \frac{1}{i} x_{i}$ does not $\rho$-converge in $X$, however $\left(\frac{1}{i}\right) \in c_{0}$, this implies that $c_{0}$ is not contained in $S_{\rho}\left(\sum x_{i}\right)$, which contradicts Corollary 1.

Theorem 2 has a counterpart for the weak topology in $X$. For an ideal $\mathcal{I}$ we can define the weak- $\mathcal{I}$ convergence space associated to a wuc series $\sum_{i} x_{i}$ :

$$
S_{w \mathcal{I}}=\left\{\left(a_{i}\right) \in \ell^{\infty},: \sum_{i} a_{i} x_{i} \text { is weakly- } \mathcal{I} \text {-convergent }\right\} .
$$

The proof of the following results mimics the proof of Theorem 2, we include it for the sake of completeness.

Theorem 4. Let $\mathcal{I}$ be a non-trivial and regular ideal. On a real Banach space $X$, the following conditions are equivalents:

1. $\sum_{i} x_{i}$ is a wuc series.

2. $S_{w \mathcal{I}}\left(\sum_{i} x_{i}\right)$ is complete.

3. $c_{0} \subset S_{w \mathcal{I}}\left(\sum_{i} x_{i}\right)$.

Proof. Let us show that $(1) \Rightarrow(2)$. Since $\sum x_{i}$ is wuc, the following supremum is finite:

$$
H=\sup \left\{\left\|\sum_{i=1}^{n} a_{i} x_{i}\right\|:\left|a_{i}\right| \leq 1,1 \leq i \leq n, n \in \mathbb{N}\right\}<+\infty .
$$


Let $\left(a^{m}\right)_{m} \subset S_{w \mathcal{I}}\left(\sum_{i} x_{i}\right)$ such that $\lim _{m}\left\|a^{m}-a^{0}\right\|_{\infty}=0$, with $a^{0} \in \ell^{\infty}$. We wish to prove that $a^{0} \in S_{w \mathcal{I}}\left(\sum_{i} x_{i}\right)$

Since $a^{m} \in S_{w \mathcal{I}}\left(\sum_{i} x_{i}\right)$ there exists $z_{m} \in X$ such that $\sum_{i=1}^{n} a_{i}^{m} f\left(x_{i}\right) \stackrel{\mathcal{I}}{\longrightarrow} f\left(z_{m}\right)$ for all $f \in X^{*}$. We claim that $\left(z_{m}\right)$ is a Cauchy-sequence (convergence in norm). Indeed, let us fix $\varepsilon>0$. There exists $k_{0} \in \mathbb{N}$ such that $\left\|a^{m}-a^{0}\right\|_{\infty}<\frac{\varepsilon}{4 H}$, for all $m \geq k_{0}$. Let us suppose that $p, q \in \mathbb{N}$ satisfy $p, q \geq k_{0}$. Let $f_{0} \in S_{X^{*}}$ (here $S_{X^{*}}$ denotes the unit sphere in $X^{*}$ ) such that $\left\|z_{p}-z_{q}\right\|=f_{0}\left(z_{p}-z_{q}\right)$. The vectors $f_{0}\left(z_{p}\right)$ and $f_{0}\left(z_{q}\right)$ are respectively $\mathcal{I}$-limits of the sequences $f_{0}\left(S_{n}^{p}\right)=\sum_{i=1}^{n} a_{i}^{p} f_{0}\left(x_{i}\right)$ and $f_{0}\left(S_{n}^{q}\right)=\sum_{i=1}^{n} a_{i}^{q} f_{0}\left(x_{i}\right)$. Therefore, since $\mathcal{I}$ is non-trivial, there exist integers $n_{k}$ (in fact there exists an increasing sequence $\left(n_{k}\right)$ ) such that

$$
\left|\left(\sum_{i=1}^{n_{k}}\left(a_{i}^{p}-a_{i}^{q}\right) f_{0}\left(x_{i}\right)\right)-f_{0}\left(z_{p}-z_{q}\right)\right|<\frac{\varepsilon}{2} .
$$

We can suppose without loss that $\left\|a^{p}-a^{q}\right\| \neq 0$ otherwise there exists a subsequence of $\left(a^{m}\right)$ which is constant, therefore we have trivially that $a^{0} \in S_{w \mathcal{I}}\left(\sum_{i} x_{i}\right)$. Let us observe that for all $n \in \mathbb{N}$

$$
\frac{1}{\left\|a^{p}-a^{q}\right\|}\left\|\sum_{i=1}^{n}\left(a_{i}^{p}-a_{i}^{q}\right) x_{i}\right\| \leq H,
$$

therefore,

$$
\left|\sum_{i=1}^{n}\left(a_{i}^{p}-a_{i}^{q}\right) f_{0}\left(x_{i}\right)\right| \leq \frac{\varepsilon}{2}
$$

Hence, using the equation $\left\|z_{p}-z_{q}\right\|=f_{0}\left(z_{p}-z_{q}\right)$, the inequalities (2) and (4), and the triangular inequality we get $\left\|z^{p}-z^{q}\right\| \leq \varepsilon$ for all $p, q \geq k_{0}$ as desired.

Using the same argument used when obtaining inequalities (3) and (4), if $\left\|a^{m}-a^{0}\right\| \leq \frac{\varepsilon}{4 H}$ then for all $f \in S_{X^{*}}$ and for all $n \in \mathbb{N}$ we get that

$$
\left|f\left(S_{n}^{m}\right)-f\left(S_{n}^{0}\right)\right|<\frac{\varepsilon}{2}
$$

Finally, since $X$ is complete, let $z_{0}$ be the limit of the Cauchy sequence $\left(z_{m}\right)$. We will show that for every $f \in S_{x^{*}}$, we have that $f\left(S_{n}^{0}\right) \stackrel{\mathcal{I}}{\longrightarrow} f\left(z_{0}\right)$. Indeed, let $\varepsilon>0$, we wish to show that

$$
A=\left\{n \in \mathbb{N}:\left|f\left(S_{n}^{0}\right)-f\left(z_{0}\right)\right|>\varepsilon\right\} \in \mathcal{I} .
$$

By Equation (5) if $\left\|z_{m}-z_{0}\right\| \leq \frac{\varepsilon}{16 \mathrm{H}}$ we obtain that that $\left|f\left(S_{n}^{m}\right)-f\left(S_{n}^{0}\right)\right| \leq \varepsilon / 4$ for all $n \in \mathbb{N}$. Now, if $0<\delta<\frac{\varepsilon}{2}$, since $f\left(S_{n}^{m}\right) \stackrel{\mathcal{I}}{\longrightarrow} f\left(z_{m}\right)$ the subset

$$
B=\left\{n \in \mathbb{N}:\left|f\left(S_{n}^{m}\right)-f\left(z_{m}\right)\right|>\delta\right\} \in \mathcal{I} .
$$

Thus, if $n \in A$ then:

$$
\begin{aligned}
\left|f\left(S_{n}^{m}\right)-f\left(z_{m}\right)\right| & =\left|f\left(S_{n}^{m}\right)-f\left(S_{n}^{0}\right)+f\left(S_{n}^{0}\right)-f\left(S_{n}^{0}\right)-f\left(z_{0}\right)+f\left(z_{0}\right)\right| \\
& \geq\left|f\left(S_{n}^{0}\right)-f\left(z_{0}\right)\right|-\mid f\left(S_{n}^{m}-f\left(S_{n}^{0}\right)|-| f\left(z_{0}\right)-f\left(z_{m}\right) \mid\right. \\
& \geq \varepsilon-\frac{\varepsilon}{4}-\frac{\varepsilon}{4}=\frac{\varepsilon}{2}>\delta
\end{aligned}
$$

that is, we have show that $A \subset B$, that is, we get Statement (6), and this proves that $a^{0} \in S_{w \mathcal{I}}\left(\sum_{i} x_{i}\right)$, as we desired. 
Now, let us observe that if $S_{w \mathcal{I}}\left(\sum_{i} x_{i}\right)$ is a complete space, it contains the space of eventually zero sequences $c_{00}$ and therefore we get $(2) \Rightarrow(3)$. Finally, (3) $\Rightarrow(1)$ follows by a similar argument like in Theorem 2, and this finishes the proof.

Let $\rho$ be a summability method in $\mathbb{R}$. We say that a sequence $\left(x_{n}\right)$ in a normed space $X$ is $w \rho$-convergent to $L \in X$ if for every $f \in X^{*}$, we have that $f(L)$ is the $\rho$-limit of the sequence $\left(f\left(x_{n}\right)\right)$.

$$
S_{w \rho}\left(\sum_{i} x_{i}\right)=\left\{\left(a_{i}\right) \in \ell^{\infty}: \sum_{i=1}^{n} a_{i} x_{i} \text { is } w \rho \text {-convergent }\right\} .
$$

As a consequence of Theorem 4 we obtain the following result.

Corollary 3. Let $\rho$ be a natural summability method, then on a Banach space, the following conditions are equivalent:

1. $\sum_{i} x_{i}$ is a wuc series.

2. $S_{w o}\left(\sum_{i} x_{i}\right)$ is complete.

3. $c_{0} \subset S_{w \rho}\left(\sum_{i} x_{i}\right)$.

Finally, let us consider a summability method $\rho: \mathbb{R}^{\mathbb{N}} \rightarrow \mathbb{R} \cup\{ \pm \infty\}$. We say that $\rho$ is regular in its full sense, if for each $\left(x_{n}\right)_{n}$ such that $\lim _{n \rightarrow \infty} x_{n}=x, x \in \mathbb{R} \cup\{ \pm \infty\}$, we have that $x_{n} \stackrel{\rho}{\longrightarrow} x$.

The summability method $\rho$ defines a natural summability method on the dual of a normed space $X$, we say that a sequence $\left(f_{j}\right) \subset X^{*}$ is said to be $w^{*} \rho$ convergent to $f \in X^{*}$ (in brief $f_{k} \stackrel{w^{*} \rho}{\longrightarrow} f$ ) if for any $x \in X$ we have that $f_{j}(x) \stackrel{\rho}{\longrightarrow} f(x)$. Thus $\rho$ defines the following subspaces of $\ell^{\infty}$.

$$
S_{w^{*} \rho}\left(\sum f_{i}\right)=\left\{\left(a_{i}\right) \in \ell^{\infty}: \sum_{i=1}^{n} a_{i} f_{i} \text { is } w^{*} \rho \text {-convergent }\right\} .
$$

The following result characterizes when a series in the dual space $X^{*}$ is wuc, when a Banach space $X$ is barrelled.

Theorem 5. Let $\rho$ be a full regular summability method in $\mathbb{R}$. Let $\sum f_{i}$ be a series in the dual space $X^{*}$ of a real normed space $X$. Let us consider the following conditions:

1. $\sum_{i} f_{i}$ is wuc.

2. $S_{w^{*} \rho}\left(\sum f_{i}\right)=\ell^{\infty}$.

3. For all $x \in X$ and $M \subset \mathbb{N}$, the series $\sum_{i \in M} f_{i}(x)$ is $\rho$-convergent.

Then $(1) \Rightarrow(2) \Rightarrow(3)$. Additionally if $X$ is barrelled, then (3) $\Rightarrow(1)$.

Proof. Let $\left(a_{i}\right) \in \ell^{\infty}$, since the unit ball of $X^{*}$ is weakly-* compact, then the series $\sum_{i=1}^{\infty} a_{i} f_{i}(x)$ is weakly convergent in $X^{*}$. Hence, there exists $f \in X^{*}$ such that $\sum_{i=1}^{n} a_{i} f_{i} \stackrel{w *}{\longrightarrow} f$, in particular for all $x \in X, \sum_{i=1}^{\infty} a_{i} f_{i}(x)=f(x)$. Since $\rho$ is regular we have that $\sum_{i=1}^{n} a_{i} f_{i}(x) \stackrel{\rho}{\longrightarrow} f(x)$ which implies that $\sum_{i=1}^{n} a_{i} f_{i} \stackrel{w^{*} \rho}{\longrightarrow} f$, hence we get that $\left(a_{i}\right) \in S_{w^{*} \rho}\left(\sum f_{i}\right)$. The implication $(2) \Rightarrow$ (3) follows directly. Finally if $X$ is barrelled, to prove (3) $\Rightarrow(1)$, it is sufficient to show that the subset

$$
E=\left\{\sum_{i=1}^{n} a_{i} f_{i}: n \in \mathbb{N},\left|a_{i}\right| \leq 1\right\}
$$

is pointwise bounded. Suppose on the contrary that there exists $x_{0}$ such that $\sum_{i=1}^{\infty}\left|f_{i}\left(x_{0}\right)\right|=\infty$. Then we can decompose $M^{+}=\left\{i \in \mathbb{N}: f_{i}\left(x_{0}\right)>0\right\}$ and $M^{-}=\left\{i: f_{i}\left(x_{0}\right)<0\right\}$. Then either $\sum_{i \in M^{+}} f_{i}\left(x_{0}\right)$ or $\sum_{i \in M^{-}}\left(-f_{i}\left(x_{0}\right)\right)$ diverges to infinity, since $\rho$ is full regular, we have that 
$\sum_{i=1, i \in M^{+}}^{n} f_{i}\left(x_{0}\right)$ or $\sum_{i=1, i \in M^{-}}^{n}\left(-f_{i}\left(x_{0}\right)\right)$ does not $\rho$ converges to any element, which is a contradiction with (3).

\section{Some Applications}

In this section we will see how the results on Section 3 unify the known results and how they can help us to anwer some open questions. Given a convergence method $\rho$ for series in a normed space $X$ we define abstractly the $\rho$-sequence space associated to a wuc-series $\sum_{i} x_{i}$ as follows

$$
S_{\rho}\left(\sum x_{i}\right)=\left\{\left(a_{i}\right) \in \ell^{\infty}: \sum_{i=1}^{n} a_{i} x_{i} \text { is } \rho \text {-convergent in } X\right\} .
$$

Let us consider the following statements.

Statement A. For a Banach space $X$, the following conditions are equivalent:

(i) $\sum x_{i}$ is wuc.

(ii) The subspace $S_{\rho}\left(\sum_{i} x_{i}\right)$ is closed in $\ell^{\infty}$.

(iii) $c_{0} \subset S_{\rho}\left(\sum_{i} x_{i}\right)$.

Statement B. A normed space $X$ is complete if and only if $S_{\rho}\left(\sum x_{i}\right)$ is closed in $\ell^{\infty}$ for each wuc series $\sum x_{i}$

Statement C. Let $\sum x_{i}^{*}$ be a series in the dual space $X^{*}$ of a normed space $X$. In the following conditions we have $(i) \Rightarrow($ ii $) \Rightarrow$ (iii). If $X$ is barrelled, then $($ iii $) \Rightarrow(i)$.

(i) $\sum_{i} x_{i}^{*}$ is wuc.

(ii) $S_{w^{*} \rho}\left(\sum x_{i}^{*}\right)=\left\{\left(a_{i}\right) \in \ell^{\infty},: \sum_{i=1}^{n} a_{i} x^{*}\right.$ is $\sigma\left(X^{*}, X\right)$ - $\rho$-convergent $\}=\ell^{\infty}$.

(iii) For all $x \in X$ and $M \subset \mathbb{N}$, the series $\sum_{i \in M} x_{i}^{*}$ is $\rho$-convergent.

Norm Topology. It was established in [6], Theorems 2.1 and 2.2, that Statements A and B are valid when $\rho$ is the norm convergence in $X$. When $\rho$ is the weak convergence in $X$, this was obtained in [6], Lemma 3.1, Theorems 3.2 and 3.4. Statement C, was also proved in [6] Theorem 4.1. Let us mention that all results in [6] can be obtained from the results in Section 3, if we take the ideal $\mathcal{I}_{\text {fin }}$ of the finite subsets of $\mathbb{N}$.

Statistical Convergence and Strong $\mathbf{w}^{p}$-Cesàro Convergence. Statements $\mathrm{A}-\mathrm{C}$ were obtained for $\rho=$ the statistical convergence in [11] and more recently if $\rho=$ strong ${ }^{p}{ }^{p}$-convergence in $X$ ([12] Theorem 3.1, and Theorem 3.4 (only for $p \geq 1$ ). A sequence $\left(x_{n}\right)$ in a normed space $X$ is said to be weakly-w ${ }^{p}$ convergent to $L \in X$ if for every $f \in X^{*}$ we have $\lim _{n \rightarrow \infty} \frac{\sum_{i=1}^{n}\left|f\left(x_{i}\right)-f(L)\right|^{p}}{n}=0$. The statements A-C were also obtained for the weak-w ${ }^{p}$ convergence (see [12] Theorem 4.1). Let us remark that the methods of proof in [12] cannot cover Statement B for $0<p<1$, this was an open question posed at [12]. Let us denote by $d$ the usual density defined on the subsets of natural numbers. It is well known that the statistical convergence is a kind of ideal convergence, where the ideal is defined by $\mathcal{I}_{d}=\{A \subset \mathbb{N},: d(A)=0\}$. On the other hand, it is well known by a result by J. Connor ([16]) that the strong Cesàro convergence is naturally defined, that is, there is an ideal (in this case the ideal is $\mathcal{I}_{d}$ ) such that on the realm of all bounded sequences, the Strong Cesàro convergence and the $\mathcal{I}_{d}$-convergence are equivalents. Moreover, if a sequence converges $\mathrm{w}^{p}$ strong to some limit $L$ then the sequence also converges statistically to the same limit. Therefore all results in [11,12] can be obtained from the results in Section 3. Moreover, we solve the questions in [12] proving Theorem 3.4 for $0<p<1$.

$A$-Statistical Convergence and $A$-Strong Convergence. Let us consider a matrix $A=$ $\left(\alpha_{i j}\right)_{(i, j) \in \mathbb{N} \times \mathbb{N}}$ with non-negative entries. A sequence $\left(x_{i}\right)$ in the space $X$ is said to be $A$-strong summable to $L \in X$, if $\lim _{n} \sum_{j} \alpha_{n j}\left\|x_{j}-L\right\|=0$. Also, the sequence $\left(x_{j}\right)$ is said to be $A$-statistically convergent to $L$ if for any $\varepsilon>0$

$$
\lim _{n \rightarrow \infty} \sum_{\substack{j \\\left\|x_{j}-L\right\| \geq \varepsilon}} \alpha_{n j}=0
$$


Both concepts were introduced by Connor in [13] as a natural extension of the strong-Cesàro convergence and the classical statistical convergence. It was proved that in the realm of the bounded sequences both notions are equivalent. Moreover, if $\left(x_{n}\right)$ is a sequence that $A$-strong converges to $L$ then $L$ is also the $A$ statistical limit of $\left(x_{n}\right)_{n}$. It was posed in [12] that if Statements A-C are valid when $\rho$ is the $A$-statistical convergence or the $A$-strong convergence (also it is an open question when $\rho$ is the weak $A$-statistical convergence or the $A$-strong convergence). It is well known that given a non-negative matrix $A$ and a subset $B \subset \mathbb{N}$, we can define the $A$-density of $B d^{A}(B)=\lim _{i \rightarrow \infty} \sum_{k \in B} a_{i k}$ whenever this limit exists. And by means of the density $d^{A}(\cdot)$ it is possible to define the ideal $\mathcal{I}(A)=\left\{B \subset \mathcal{N}: d^{A}(B)=0\right\}$. Therefore if $A$ is regular, $A$ defines a regular non-trivial ideal $\mathcal{I}(A)$ and the $\mathcal{I}(A)$-convergence is equivalent to the $A$-statistical convergence. As a consequence the $A$-strong convergence is a natural convergence method (in the sense of Definition 1). Thus, the results in Section 3 answer the questions posed in [12].

$f$-Statistical Convergence and $f$-Strong Cesàro Convergence. Let us recall that $f: \mathbb{R}^{+} \rightarrow \mathbb{R}^{+}$ is said to be a modulus function if it satisfies:

1. $f(x)=0$ if and only if $x=0$.

2. $f(x+y) \leq f(x)+f(y)$ for every $x, y \in \mathbb{R}^{+}$.

3. $f$ is increasing.

4. $f$ is continuous from the right at 0 .

A sequence $\left(x_{n}\right)$ is said to be $f$-strong Cesàro convergent to $L$ if

$$
\lim _{n \rightarrow \infty} \frac{f\left(\sum_{k=1}^{n}\left\|x_{k}-L\right\|\right)}{f(n)}=0
$$

In [17] the notion of $f$-statistical convergence was introduced. A sequence $\left(x_{n}\right)$ is said to be $f$-statistically convergent to $L$ if for every $\varepsilon>0$.

$$
\lim _{n \rightarrow \infty} \frac{f\left(\#\left\{k \leq n:\left\|x_{k}-L\right\|>\varepsilon\right\}\right)}{f(n)}=0 .
$$

With the $f$-statistical convergence emerges a new concept of density ( $f$-density) of subsets of natural numbers. Namely, if $A \subset \mathbb{N}$ the $f$-density of $A$ is defined by

$$
d_{f}(A)=\lim _{n \rightarrow \infty} \frac{f(\#\{k \leq n: k \in A\})}{f(n)}
$$

when this limit exists. In was proved in [18] that for many modulus functions $f$ (in fact, these modulus functions are characterized), the density $d_{f}$ does not define the usual statistical convergence. The $f$-statistical convergence is a special case of ideal convergence. Namely the ideal which defines the $f$-statistical convergence is $\mathcal{I}_{f}=\left\{B \subset \mathbb{N},: d_{f}(B)=0\right\}$. Moreover, it was proved in [18] that the $f$-strong Cesàro convergence is a natural summability method. Therefore the results in Section 3 can be used to obtain Statements $\mathrm{A}-\mathrm{C}$ for the $f$-statistical convergence, $f$-strong Cesàro convergence and its weak versions.

Erdős-Ulam Convergence. Let $p=\left(p_{n}\right)$ be a sequence of non-negative reals with $s_{i}=\sum_{k=1}^{i} p_{k} \neq$ 0 for all $i \in \mathbb{N}, \lim _{i \rightarrow \infty} s_{i}=+\infty$ and $\lim _{i \rightarrow \infty} \frac{p_{i}}{s_{i}}=0$. The family

$$
\mathcal{E} \mathcal{U}_{p}=\left\{A \subset \mathbb{N},: \lim _{n \rightarrow \infty} \frac{\sum_{i \in A, i \leq n} p_{i}}{\sum_{i \leq n} p_{i}}=0\right\}
$$

is called the Erdôs-Ulam ideal generated by $p$. The Erdős-Ulam ideal induces a regular summability method. And for this summability method Statements A-C can be obtained using the results in Section 3. 
Author Contributions: All authors have contributed to all results in the manuscript. F.L.-S. contributed mostly in Theorem 2, F.J.P.-F. contributed mostly to Theorem 4, M.d.P.R.d.l.R. contributed mostly in Theorem 5, A.S. contributed mostly in Theorem 3.

Funding: The authors are supported by Ministerio de Ciencia, Innovación y Universidades under PGC2018-101514-B-100, by Junta de Andalucía FQM-257 and Plan Propio de la Universidad de Cádiz.

Acknowledgments: The authors are indebted to Cihan Orhan for providing us some information about $\mathcal{I}$-convergence.

Conflicts of Interest: The authors declare no conflicts of interest.

\section{References}

1. Zygmund, A. Trigonometrical Series; Dover Publications: New York, NY, USA, 1955; p. vii+329.

2. Boos, J. Classical and Modern Methods in Summability; Oxford Mathematical Monographs; Oxford University Press: Oxford, UK, 2000; p. xiv+586.

3. Mursaleen, M. Applied Summability Methods; SpringerBriefs in Mathematics; Springer: Cham, Switzerland, 2014; p. x+124.

4. Kolk, E. The statistical convergence in Banach spaces. Tartu Ül. Toimetised 1991, 928, 41-52.

5. Connor, J.; Ganichev, M.; Kadets, V. A characterization of Banach spaces with separable duals via weak statistical convergence. J. Math. Anal. Appl. 2000, 244, 251-261. doi:10.1006/jmaa.2000.6725. [CrossRef]

6. Pérez-Fernández, F.J.; Benítez-Trujillo, F.; Aizpuru, A. Characterizations of completeness of normed spaces through weakly unconditionally Cauchy series. Czechoslov. Math. J. 2000, 50, 889-896. [CrossRef]

7. Aizpuru, A.; Armario, R.; Pérez-Fernández, F.J. Almost summability and unconditionally Cauchy series. Bull. Belg. Math. Soc. Simon Stevin 2008, 15, 635-644. [CrossRef]

8. Aizpuru, A.; Gutiérrez-Dávila, A.; Sala, A. Unconditionally Cauchy series and Cesàro summability. J. Math . Anal. Appl. 2006, 324, 39-48. [CrossRef]

9. Aizpuru, A.; Pérez-Eslava, C.; Seoane-Sepúlveda, J.B. Matrix summability methods and weakly unconditionally Cauchy series. Rocky Mt. J. Math. 2009, 39, 367-380. [CrossRef]

10. Aizpuru, A.; Pérez-Fernández, F.J. Sequence spaces associated to a series in a Banach space (sequence spaces associated to a series). Indian J. Pure Appl. Math. 2002, 33, 1317-1329.

11. Aizpuru, A.; Nicasio-Llach, M. Spaces of sequences defined by the statistical convergence. Studia Sci. Math. Hung. 2008, 45, 519-529. [CrossRef]

12. León-Saavedra, F.; Moreno-Pulido, S.; Sala, A. Completeness of a normed space via strong p-Cesàro convergence. Filomat 2019, in press.

13. Connor, J. On strong matrix summability with respect to a modulus and statistical convergence. Can. Math. Bull. 1989, 32, 194-198. [CrossRef]

14. Fridy, J.A. On statistical convergence. Analysis 1985, 5, 301-313. [CrossRef]

15. Dems, K. On I-Cauchy sequences. Real Anal. Exch. 2004, 30, 123-128. [CrossRef]

16. Connor, J.S. The statistical and strong p-Cesàro convergence of sequences. Analysis 1988, 8, 47-63. [CrossRef]

17. Aizpuru, A.; Listán-García, M.C.; Rambla-Barreno, F. Double density by moduli and statistical convergence. Bull. Belg. Math. Soc. Simon Stevin 2012, 19, 663-673. [CrossRef]

18. León-Saavedra, F.; Carmen, L.G.M.; Pérez-Fernández, F.; Romero de la Rosa, M.d.P. On statistical convergence and Strong Cesàro convergence by moduli. J. Inequal. Appl. 2019, in press.

(C) 2019 by the authors. Licensee MDPI, Basel, Switzerland. This article is an open access article distributed under the terms and conditions of the Creative Commons Attribution (CC BY) license (http:/ / creativecommons.org/licenses/by/4.0/). 